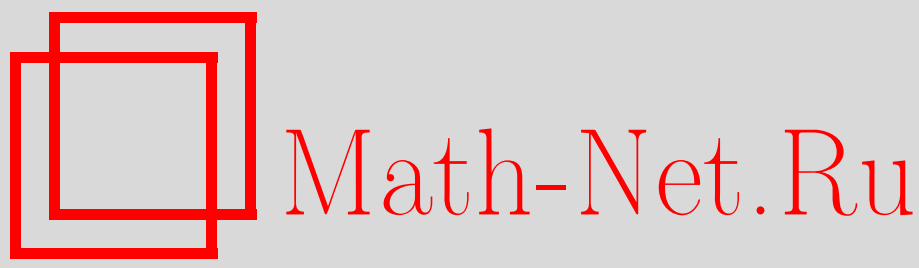

В. П. Радченко, П. И. Краснощеков, А. Ф. Федотов, Контактно-стержневая модель пластического деформирования порошковых материалов, Вестн. Сам. гос. техн. ун-та. Сер. Физ.-мат. науки, 2004, выпуск 26, 102-107

DOI: https://doi.org/10.14498/vsgtu185

Использование Общероссийского математического портала Math-Net.Ru подразумевает, что вы прочитали и согласны с пользовательским соглашением

http://www . mathnet.ru/rus/agreement

Параметры загрузки:

IP: 54.198 .55 .26

26 апреля 2023 г., 14:25:59

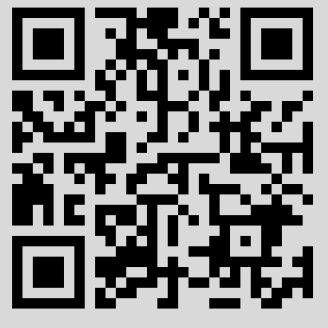




\section{КОНТАКТНО-СТЕРЖНЕВАЯ МОДЕЛЬ ПЛАСТИЧЕСКОГО ДЕФОРМИРОВАНИЯ ПОРОШКОВЫХ МАТЕРИАЛОВ}

Предложена структурная модель, учитывающая контактную природу пластического деформирования порошкового тела. Модель представляет собой хаотично ориентированную контактностержневую систему, которая испытывает однородную деформацию растяжения-сжатия. Контактно-стержневая модель удовлетворяет граничным условиям для состояния насыпной плотности и показывает хорошее соответствие с экспериментальными данными по изостатическому прессованию металлически порошков.

Континуальные теории пластического деформирования пористых и порошковых материалов строятся на результатах структурного моделирования. Макроскопические характеристики квазиоднородных несплошных тел получаются в результате осреднения локальных характеристик материала по принятому представительному объему. Поэтому способ осреднения в итоге определяет адекватность континуальных моделей механического поведения пористых и порошковых тел.

При конструировании механических моделей несплошных тел мощность диссипируемой энергии связывают только с пластической деформацией вещества (постулат В.В. Скорохода об однозначности диссипативной функции). Статистическое осреднение локальных напряжений и деформаций проводят по всему объему твердой фазы [1]. Объем, по которому выполнено осреднение силовых или кинематических параметров, находится в однородном напряженнодеформированном состоянии (НДС). Поэтому осреднение по всему объему вещества несплошного тела означает, что независимо от вида макроскопической деформации все вещество испытывает однородную деформацию растяжения-сжатия или сдвига $[1,2]$. В рамках этой модели диссипативные функции несплошного тела $D$ и его вещества с однородным НДС $D_{0}$ будут связаны зависимостью

$$
D=\rho D_{0},
$$

где $\rho$ - объемная доля твердой фазы или относительная плотность. Из (1) следует эллиптическое условие пластичности для сжимаемых пористых тел с идеально-пластической твердой фазой [3]:

$$
\sigma^{2} / 2 \psi+T^{2} / \varphi=\rho \tau_{0}^{2}
$$

где $\sigma$ - среднее напряжение; $T$ - интенсивность касательных напряжений сдвига; $\tau_{0}-$ предел текучести на сдвиг вещества твердой фазы; $\psi, \varphi$ - функции относительной плотности $\rho$, равные: $\psi=2 \rho^{3} / 3(1-\rho), \varphi=\rho^{2}$. В (2) интенсивность касательных напряжений сдвига $T$ определяется через компоненты девиатора напряжений $s_{i j}$ соотношением $T=\sqrt{0,5 s_{i j} s_{i j}}$ и отличается от используемой в [3] интенсивности касательных напряжений $\tau=\sqrt{s_{i j} s_{i j}}$. Это приводит к появлению множителя 2 перед функцией $\psi$. При допущении об однородности полей деформаций или напряжений во всем объеме твердой фазе условие пластичности, аналогичное условию (2), получается как в рамках стохастических моделей [4-7], так и при обобщении гипотезы Бельтрами на пористые тела $[8,9]$.

Условие пластичности (2) часто применяют для порошков при схемах деформирования в замкнутых объемах с преобладанием сжимающих нагрузок $[9,10]$. В отличие от пористых тел пластическая деформация порошков в состоянии насыпной плотности, когда $\rho=\rho_{0}$, начинается при произвольно малых нагрузках. Вместе с тем из (2) следует, что для состояния насыпной плотности сопротивление порошка чистому сдвигу $T$ отлично от нуля: $T=\tau_{0} \sqrt{\rho_{0} \varphi}$. Следовательно, структурная модель с осреднением локальных напряжений и скоростей деформаций по всему объему вещества не удовлетворяет граничным условиям для начального (насыпного) состояния порошкового тела. 
В ряде работ $[11,12]$ отсутствие сопротивления деформации порошков при насыпной плотности учитывается через функции пористости $\psi, \varphi$. В этом случае проводят нормировку функций $\psi$ и $\varphi$ : вместо относительной плотности $\rho$ используется параметр вида

$$
\bar{\rho}=\frac{\rho-\rho_{0}}{1-\rho_{0}},
$$

который принимает нулевое значение при $\rho=\rho_{0}$. При этом нормируется либо один [11], либо все аргументы $\rho[12]$, входящие в функции $\psi$ и $\varphi$. Такой подход носит формальноматематический характер и не имеет физического обоснования. В настоящей работе предлагается вариант структурной модели, учитывающий контактную природу пластического деформирования порошковых тел.

Пусть порошковое тело нагружено однородным полем макронапряжений $\sigma_{i j}$, скольжения частиц нет и уплотнение происходит за счет пластической деформации частиц. Выделим элементарную ячейку объемом $V$, в которую вписана деформированная частица порошка объемом $V_{0}$. Мощность диссипации порошкового тела в ячейке при однородном поле макронапряжений будет равна:

$$
D \cdot V=\int_{V_{0}} \sigma_{i j}^{\prime} e_{i j}^{\prime} d V
$$

где $D$ - удельная мощность диссипации порошкового тела; $\sigma_{i j}^{\prime}, e_{i j}^{\prime}$ - тензоры действительного неоднородного поля микроскопических напряжений и скоростей пластических деформаций в веществе ячейки. Вместо неоднородного поля напряжений $\sigma_{i j}^{\prime}$ и скоростей деформаций $e_{i j}^{\prime}$ введем эквивалентное ему однородное поле напряжений $\sigma_{i j}^{o}$ и скоростей деформаций $e_{i j}^{o}$. Пусть новое поле силовых и кинематических параметров занимает в веществе ячейки объем $V_{\alpha}$, причем $V_{\alpha} \leq V_{0}$. Условие эквивалентного перехода сформулируем в виде равенства мощности диссипации действительных неоднородных и эквивалентных им однородных полей напряжений и скоростей деформаций:

$$
\int_{V_{\alpha}} \sigma_{i j}^{o} e_{i j}^{o} d V=\int_{V_{0}} \sigma_{i j}^{\prime} e_{i j}^{\prime} d V .
$$

Подынтегральное произведение в левой части (5) представляет собой диссипативную функцию пластического вещества $D_{0}$ с однородным НДС. С учетом (5) тождественное равенство диссипативных функций ячейки порошкового тела $D$ и вещества ячейки $D_{0}$ запишется в виде

$$
D=\alpha D_{0},
$$

где $\alpha=V_{\alpha} / V$ - объемная доля вещества ячейки с однородными полями напряжений и скоростей пластических деформаций.

Рассмотрим основное энергетическое уравнение для материального элемента объемом $V_{\alpha}$ и поверхностью $S_{\alpha}$ :

$$
\int_{V_{\alpha}} \sigma_{i j}^{\mathrm{o}} e_{i j}^{\mathrm{o}} d V=\int_{S_{\alpha}} p_{i}^{\mathrm{o}} v_{i}^{\mathrm{o}} d S_{\alpha},
$$

где $p_{i}^{\circ}$ - поверхностные напряжения; $v_{i}^{o}$ - скорости перемещения. Из (7) следует, что объем $V_{\alpha}$ будет ограничен контактными площадками, на которых $p_{i}^{0} \neq 0$. Для уточнения формы контактных объемов сечением $A$, параллельным контактной площадке и удаленным от нее на расстояние $z=h$, выделим элемент частицы (рис. 1). Напряжения $p_{i}^{\circ}$ на контактной площадке площадью $S_{\text {к }}$ и нормалью $\boldsymbol{n}$ представим в виде суммы векторов нормальных $\sigma_{n}^{\circ}$ и касательных $\tau_{n}^{\circ}$ напряжений, приложенных в центре тяжести площадки. Внутри сечения $A$ находится область площадью $S_{A \alpha}$ с однородным НДС, которое характеризуется векторами нормальных $\boldsymbol{\sigma}_{n \alpha}^{\circ}$ и касательных $\tau_{n \alpha}^{\circ}$ напряжений. Векторы $\boldsymbol{\sigma}_{n \alpha}^{\circ}$ и $\tau_{n \alpha}^{\circ}$ приложены в центре тяжести площадки $S_{A \alpha}$. Для однородного НДС должны выполняться следующие условия:

$$
\sigma_{n}^{\mathrm{o}}=\sigma_{n \alpha}^{\mathrm{o}}=\text { const }, \tau_{n}^{\mathrm{o}}=\tau_{n \alpha}^{\mathrm{o}}=\text { const }
$$


Выясним, какой вид деформации частицы отвечает условиям однородности. С сечением $A$ свяжем систему декартовых координат, направив ось $z$ вдоль вектора $\sigma_{n}^{o}$, а ось $y$ - параллельно вектору $\tau_{n}^{\mathrm{o}}$. Из уравнения равновесия элемента

$$
\sum m_{x}=0, \quad \tau_{n}^{\mathrm{o}} S_{\mathrm{K}} h-\int_{S_{\alpha}} \sigma_{n \alpha} y d S=0
$$

следует, что касательные напряжения на контактной площадке $\tau_{n}^{0}$ вызывают изгиб и неоднородное распределение нормальных напряжений $\sigma_{n \alpha}$ по области $S_{A \alpha}$. Кроме того, напряжения изгиба $\sigma_{n \alpha}$ возрастают пропорционально расстоянию $h$. Поэтому при однородном НДС контактные площадки должны быть свободны от касательных напряжений и элемент частицы испытывает центральное одноосное сжатие.

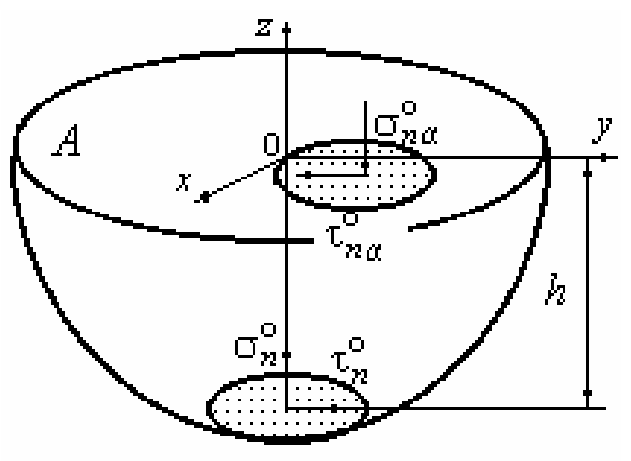

Р и с. 1. Схема деформирования частицы порошка

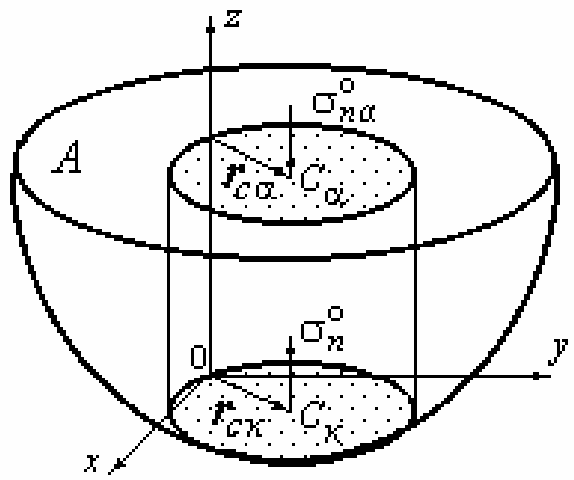

Р и с. 2. Схема одноосного сжатия частицы порошка

Для определения формы площадки $S_{A \alpha}$ с контактной площадкой $S_{\text {к }}$ свяжем систему декартовых координат, направив ось $z$ параллельно вектору $\sigma_{n}^{\circ}$. Начало координат поместим в произвольную точку границы контактной площадки (рис. 2). Положение центров тяжести площадок в принятой системе координат определяют радиус-векторы $\boldsymbol{r}_{\boldsymbol{c}}$ и $\boldsymbol{r}_{\boldsymbol{c} \alpha}$. Векторы $\boldsymbol{r}_{0 \kappa}$ и $\boldsymbol{r}_{0 \alpha}$, равные и противоположные векторам $\boldsymbol{r}_{\boldsymbol{c}}$ и $\boldsymbol{r}_{\boldsymbol{c} \alpha}$, в системе координат, связанной с центром тяжести, характеризуют форму площадок. Поэтому координаты центров тяжести, взятые с противоположным знаком, будут однозначно соответствовать координатам точек границ площадок. Координаты центра тяжести $C_{\alpha}\left(x_{\mathrm{c} \alpha}, y_{\mathrm{c} \alpha}, h\right)$ площадки $S_{A \alpha}$ и центра тяжести $C_{\mathrm{\kappa}}\left(x_{\mathrm{c \kappa}}, y_{\mathrm{c \kappa}}, 0\right)$ площадки

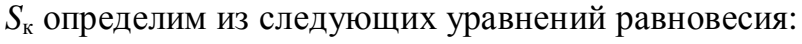

$$
\begin{aligned}
& \sum F_{k z}=0 ; \quad \sigma_{n}^{o} S_{\mathrm{\kappa}}-\sigma_{n \alpha}^{o} S_{A \alpha}=0 ; \\
& \sum m_{x}=0 ; \quad \sigma_{n}^{\mathrm{o}} S_{\mathrm{K}} y_{c \kappa}-\sigma_{n \alpha} S_{A \alpha} y_{c \alpha}=0 \text {; } \\
& \sum m_{y}=0 ; \quad \sigma_{n}^{o} S_{\kappa} x_{c \kappa}-\sigma_{n \alpha} S_{A \alpha} x_{c \alpha}=0 \text {. }
\end{aligned}
$$

Учитывая, что $\sigma_{n \alpha}^{o}=\sigma_{n}^{o}$, получим, центры тяжести площадок, имеющие одинаковые координаты $x, y$ :

$$
x_{c \alpha}=x_{c \kappa}, \quad y_{c \alpha}=y_{c \kappa}
$$

и находящиеся на общей нормали к площадкам. Аналогичным образом будут расположены любые две точки границ площадок $S_{\text {к }}$ и $S_{A \alpha}$ и, следовательно, эти площадки имеют одинаковую форму. Поэтому вещество ячейки с однородным НДС будет находиться внутри прямого цилиндра с контактной площадкой в основании.

Таким образом, в связи с локальным нагружением частицы через контактную площадку единичный объем осреднения кинематических и силовых параметров представляет собой прямой цилиндр с контактной площадкой в основании. Суммарный объем осреднения $V_{\alpha}$ будет состоять из совокупности цилиндров, построенных на всех контактных площадках частицы и имеющих общее ядро, образованное пересечением образующих цилиндров. В целом пластически деформируемое порошковое тело представляет собой хаотично ориентированную контакт- 
но-стержневую систему, которая состоит из цилиндров, контактирующих своими основаниями, и испытывает однородную деформацию растяжения-сжатия.

При установлении количественной связи между диссипативными функциями порошкового тела $D$ и вещества ячейки $D_{\alpha}$ не требуется выполнять прямое вычисление объема $V_{\alpha}$. Для этого достаточно знать долю контактного объема $\alpha$. Вероятностный анализ структурных характеристик порошковых материалов выполнен в [13, 14]. Согласно [13] доля контактного объема $\alpha$ для текущей относительной плотности $\rho$ составляет:

$$
\alpha=\rho^{2}\left(\frac{\rho-\rho_{0}}{1-\rho_{0}}\right)^{b} .
$$

Зависимость $\alpha(\rho)$, полученная в работе [14], имеет следующий вид:

$$
\alpha=\rho^{2}\left(1-\left(\frac{\ln \rho}{\ln \rho_{0}}\right)^{b}\right) .
$$

Здесь $b$ - эмпирическая константа, которая для моносферических порошков равна $b=1$. Уравнение, аналогичное уравнению (14) при $b=1$, получено в [15] из модели уплотнения за счет роста радиусов сферических частиц вокруг фиксированных центров с дальнейшим перекрытием сфер и образованием шеек контакта. В состоянии насыпной плотности $\left(\rho=\rho_{0}\right)$ объемная доля пластически деформируемого вещества $\alpha$ и диссипативная функция $D$ порошкового тела равны нулю. Тем самым обеспечивается выполнение граничных условий для насыпного состояния порошка.

Для решения краевых задач пластического деформирования порошковых тел необходимо знать определяющие соотношения между макроскопическими напряжениями $\sigma_{i j}$ и скоростями деформации $e_{i j}$. Зависимость $\sigma_{i j}\left(e_{i j}\right)$ следует из определения диссипативной функции:

$$
\sigma_{i j}=\frac{\partial D}{\partial e_{i j}} .
$$

С учетом (6) уравнение (16) запишется следующим образом:

$$
\sigma_{i j}=\alpha \frac{\partial D_{0}}{\partial e_{i j}} .
$$

Чтобы воспользоваться соотношением (17), необходимо установить функциональную зависимость между диссипативной функцией вещества $D_{0}$ и макроскопическими скоростями деформации порошкового тела $e_{i j}$ с различной реологией твердой фазы. Диссипативная функция $D_{0}$ выражается через интенсивность скоростей деформации сдвига несжимаемого вещества $W$ и его реологические константы $[15,16]$ :

$$
\text { линейно-вязкое тело: }
$$

идеально-пластическое тело:

нелинейно-вязкое тело:

$$
\begin{aligned}
& D_{0}=\frac{1}{2} \eta_{0} W^{2} ; \\
& D_{0}=\tau_{0} W ; \\
& D_{0}=\frac{1}{n+1} A W^{n+1} .
\end{aligned}
$$

В итоге задача сводится к определению зависимости интенсивности микроскопической скорости деформации сдвига $W$ от макроскопических скоростей деформации $e_{i j}$. Для определения искомой связи $W\left(e_{i j}\right)$ воспользуемся теорией вязкого и пластического течения пористых тел, изложенной в работах $[3,16,17]$. В основу теории положена следующая гипотеза: зависимость осредненной интенсивности скоростей деформации сдвига твердой фазы $W$ от макроскопических скоростей деформаций $e_{i j}$ инвариантна относительно реологических свойств твердой фазы. На основании этой гипотезы находится зависимость $W\left(e_{i j}\right)$ для линейно-вязкого тела, а затем выполняется обобщение на тела другой реологии.

Для линейно-вязкого сжимаемого материала диссипативная функция имеет вид

$$
D=\frac{1}{2}\left(\zeta e^{2}+\eta H^{2}\right),
$$


где $e$ - макроскопическая скорость объемной деформации; $H$ - интенсивность макроскопических скоростей деформации сдвига; $\zeta, \eta$ - коэффициенты объемной и сдвиговой вязкости. Коэффициенты вязкости $\zeta$ и ๆ пористого тела выражаются через коэффициент сдвиговой вязкости вещества $\eta_{0}$ и функции пористости:

$$
\zeta=2 \psi \eta_{0}, \quad \eta=\varphi \eta_{0} .
$$

После подстановки в соотношение (6) зависимостей $(18),(21)$ и (22) найдем выражение $W\left(e_{i j}\right)$ для линейно-вязкого порошкового тела:

$$
W=\frac{1}{\sqrt{\alpha}} \sqrt{2 \psi e^{2}+\varphi H^{2}} .
$$

Определяющие уравнения порошковых тел с различной реологией твердой фазы получим из (17) с учетом зависимостей (18) - (21) и (23):

линейно-вязкое тело:

$\sigma_{i j}=2 \eta_{0}\left[\left(\psi-\frac{1}{3} \varphi\right) e \delta_{i j}+\varphi e_{i j}\right]$;

идеально-пластическое тело:

$$
\sigma_{i j}=\frac{2 \tau_{0} \sqrt{\alpha}}{\sqrt{2 \psi e^{2}+\varphi H^{2}}}\left[\left(\psi-\frac{1}{3} \varphi\right) e \delta_{i j}+\varphi e_{i j}\right] \text {; }
$$

нелинейно-вязкое тело:

$$
\sigma_{i j}=2 A\left(\frac{\alpha}{2 \psi e^{2}+\varphi H^{2}}\right)^{\frac{1-n}{2}}\left[\left(\psi-\frac{1}{3} \varphi\right) e \delta_{i j}+\varphi e_{i j}\right] \text {. }
$$

Из определяющих соотношений (25) несложно получить условие пластичности порошкового тела с идеально-пластической твердой фазой:

$$
\sigma^{2} / 2 \psi+T^{2} / \varphi=\alpha \tau_{0}^{2}
$$

Следует отметить, что в определяющие уравнения для линейно-вязкого порошкового тела не входит параметр $\alpha$, который характеризует количество вещества, диссипирующего механическую энергию. Определяющие уравнения (24) идентичны уравнениям, полученным в [3, 17] при осреднении по всему объему твердой фазы, количественной мерой которого служит относительная плотность $\rho$. Таким образом, линейно-вязкое сжимаемое тело оказалось инвариантно к способу осреднению локальных напряжений и скоростей деформаций по объему вещества. Этот результат следует связать с квадратичной формой диссипативной функции линейновязкого тела. Для идеально-пластического и нелинейно-вязкого порошка, находящегося в состоянии насыпной плотности, когда $\alpha=0$, деформирующие напряжения равны нулю. Так как для порошкового тела диссипация имеет место при $\alpha>0$, то уравнение (24) можно применять только при плотности, большей, чем насыпная плотность $\rho_{0}$.

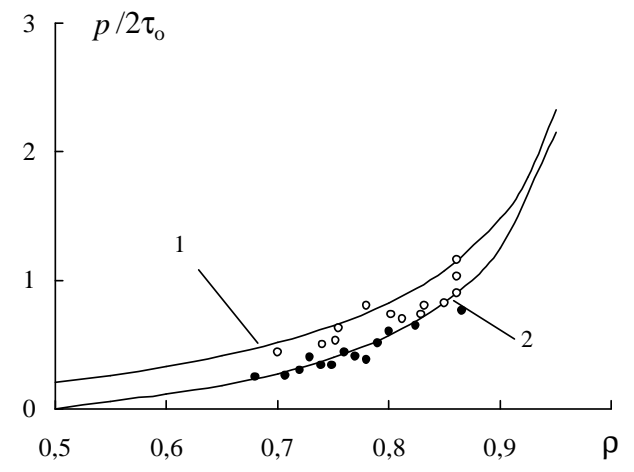

Р и с. 3. Зависимость относительного давления изостатического прессования от относительной плотности порошков:

1 - расчет по (2); 2 - расчет по (27);

эксперимент: • - олово; о - свинец

В качестве экспериментальной проверки предложенной структурной модели пластически деформируемого порошкового тела рассмотрим процесс изостатического прессования металлических порошков. На рис. 3 приведены опытные данные по уплотнению при изостатическом прессовании сферических порошков свинца и олова, взятые из работы [18]. На этом же рисунке представлены зависимости между относительной плотностью $\rho$ порошка и относительным давлением $p / 2 \tau_{0}$, рассчитанные по условиям пластичности (2) и (25).

Для расчета объемной доли пластически деформируемого вещества $\alpha$ в (25) использовалась зависимость (14). Насыпная плотность $\rho_{0}$ принималась равной $\rho_{0}=0,5$ (получено экстраполяцией опытных данных для олова) и значение показателя степени $b=1$. Из рис. 3 видно, что наилучшее соответствие расчетных и экспериментальных данных во всем диапазоне изменения плотности показывает контактностержневая модель и связанное с ней условие пластичности (27). Расхождение расчетных и экспериментальных данных для порошка свинца следует связать с более низкой, чем $\rho_{0}=0,5$, 
насыпной плотностью этого материала. Величина давления при уплотнении порошкового тела пропорциональна объемной доле пластически деформируемого вещества. Структурные модели, из которых следуют уравнения (2) и (27), не учитывают локализацию пластического течения при контактном взаимодействии частиц и предполагают пластическое деформирование всего объема вещества. Поэтому расчет по уравнениям (2) и (27) показывает более высокие значения относительного давления по сравнению с экспериментом. Соответственно осреднение микроскопических параметров по всему объему твердой фазы дает верхнюю оценку, а осреднение в пределах контактных объемов частиц - нижнюю оценку макроскопических свойств порошкового материала.

\section{БИБЛИОГРАФИЧЕСКИЙ СПИСОК}

1. Скороход В.В. Реологические основы теории спекания. К.: Наукова думка, 1972. 152 с.

2. Ковальченко M.C. Теоретические основы горячей обработки пористых материалов давлением. К.: Наукова думка, 1980. $240 \mathrm{c}$

3. Скороход В.В., Штерн М.Б., Мартынова И.Ф. Теория нелинейно-вязкого и пластического поведения пористых материалов // Порошковая металлургия. 1987. № 8. С. 23-30.

4. Смыслов А.Ю. К теории пластичности пористых сред // Изв. вузов. Машиностроение. 1980. № 4. С. 107-110.

5. Механика композитных материалов и элементов конструкций. Т. 1. Механика материалов. К.: Наукова думка, 1982. $368 \mathrm{c}$.

6. Сараев Л.А. Моделирование макроскопических пластических свойств многокомпонентных композиционных материалов. Самара: Самар. гос. ун-т, 2000. $183 \mathrm{c}$.

7. Буряченко В.А., Липанов А.М., Кожевникова Ю.Г. Уравнения вязкопластического деформирования упрочняющихся пористых сред. // Формирование и свойства высокодисперсных систем. Межвуз. сб. науч. трудов. Л.: ЛТИ им. Ленсовета, 1989. С. 140-149.

8. Скороход В.В., Тучинский Л.И. Условие пластичности пористых тел // Порошковая металлургия. 1978. № 11. C. $83-87$.

9. Григорьев А.К., Рудской А.И. Деформация и уплотнение порошковых материалов. М.: Металлургия, 1992.192 с.

10. Власов А.В. Моделирование процессов прессования порошков в капсулах с учетом проскальзывания на границах сред // Вестник МГТУ. Сер. Машиностроение. 1998. № 3. С. 87-92.

11. Каташинский В.Л., Штерн М.Б. Напряженно-деформированное состояние прокатываемого порошка в зоне уплотнения // Порошковая металлургия. 1983. № 11. С. 17-21.

12. Гун Г.Я., Стебунов С.А., Ганелин Д.Ю., Фролов А.А. Моделирование на ЭВМ и исследование процесса прокатки пористых материалов // Порошковая металлургия. 1983. № 11. С. 21-26.

13. Бальшин М.Ю. Научные основы порошковой металлургии и металлургии волокна. М.: Металлургия, 1972. $336 \mathrm{c}$.

14. Жданович Г.Н. Теория прессования металлических порошков. М.: Металлургия, 1969. 262 с.

15. Стоев П.И., Папиров И.И., Тихинский Г.Ф., Васильев А.А. Диаграммы изостатического прессования бериллия // Физика металлов и металловедение. 1994. Т. 78. Вып. 1. С. 9-19.

16. Штерн М.Б. Эквивалентные деформации и напряжения порошковых материалов. II. Связь эквивалентной деформации пористых тел с макроскопическими деформациями // Порошковая металлургия. 1987. № 2. С. 13-18.

17. Скороход В.В., Олевский Е.А., Штерн М.Б. Континуальная теория спекания. І. Феноменологическая модель. Анализ влияния внешних силовых воздействий на кинетику спекания. // Порошковая металлургия. 1993. № 1. C. $22-27$.

18. Лаптев А.М., Подлесный С.В. Расчет силовых характеристик процесса уплотнения сферических порошков // Порошковая металлургия. 1985. № 12. С. 11-17.

Поступила 14.01.2004 2. 